\title{
An advanced refractory breast cancer patient with human epidermal growth factor receptor 2 discordance: a case report
}

\author{
Yijia Hua ${ }^{1 \#}$, Chunxiao Sun ${ }^{1 \#}$, Fan Yang ${ }^{1 \#}$, Yiqi Yang ${ }^{1}$, Shengnan Bao ${ }^{1}$, Xueqi Yan ${ }^{1}$, Tianyu Zeng ${ }^{1}$, Wei Li $^{1}$, \\ Yongmei Yin ${ }^{1,2}$ \\ ${ }^{1}$ Department of Oncology, The First Affiliated Hospital of Nanjing Medical University, Nanjing, China; ${ }^{2}$ Jiangsu Key Lab of Cancer Biomarkers, \\ Prevention and Treatment, Collaborative Innovation Center for Personalized Cancer Medicine, Nanjing Medical University, Nanjing, China \\ \#These authors equally contributed to this work. \\ Correspondence to: Yongmei Yin; Wei Li. The First Affiliated Hospital of Nanjing Medical University, 300 Guangzhou Road, Nanjing 210029 , China. \\ Email: ymyin@njmu.edu.cn; real.lw@163.com.
}

\begin{abstract}
Breast cancer is heterogeneous at the molecular level, and receptor discordance has frequently been observed in breast cancer patients during treatment. Patients with human epidermal growth factor receptor 2 (HER2) discordance are reported to have poor survival, but guidelines for the treatment of these patients are still being explored. In this case report, we will share our experience in treating a hormone receptor-positive breast cancer patient with HER2 discordance. This patient was first diagnosed as Luminal B-like HER2+, and had previously undergone several lines of anti-HER2 therapy with limited effectiveness. Pathological reassessment of the patient's later metastasis showed Luminal B-like HER2-. Next-generation sequencing (NGS) testing was performed on the patient's surgical samples, and she was prescribed a therapy consisting of palbociclib and exemestane in December, 2018. This patient was subsequently followed up and her progression-free survival (PFS) has reached 16 months. This passage highlights the importance of pathological reassessment and standard treatments. In the absence of any clinical data on the usage of cyclindependent kinase inhibitors (CDKIs) in patients with HER2 discordance, we predict the potential role of palbociclib in the treatment hormone receptor-positive breast cancer patients with HER2 discordance.
\end{abstract}

Keywords: Human epidermal growth factor receptor 2 discordance (HER2 discordance); palbociclib; disease progression; precise medicine; case report

Received: 28 February 2020; Accepted: 16 June 2020; Published: 30 July 2020.

doi: $10.21037 /$ tbcr-20-3

View this article at: http://dx.doi.org/10.21037/tbcr-20-3

\section{Introduction}

Breast cancer is the most common malignancy among women globally. According to the expression status of receptors including hormone receptors and human epidermal growth factor receptor 2 (HER2), as well as the proliferation marker Ki67, breast cancer can be classified into five molecular subtypes: Luminal A-like, Luminal B-like HER2-, Luminal B-like HER2+, HER2-enriched (non-luminal), and triple-negative breast cancer (1). Continuous exploration of breast cancer has facilitated the discovery of specific targeted drugs or therapies for the different subtypes of breast cancer, which have led to improvements in prognosis and the effectiveness of treatment. Nevertheless, the majority of patients still experience disease progression eventually. For patients with advanced refractory breast cancer, it is imperative that second-time biopsy is performed (2); however, discordance at the molecular level, such as the loss of estrogen receptor status or change of HER2 status, is often observed, which brings challenges for clinical treatment (3). In particular, the impact of HER2 discordance on the treatment and prognosis of breast cancer patients has attracted considerable interest. Some retrospective studies have shown that HER2 discordance is involved with disease progression, drug resistance, and poor prognosis $(3,4)$. However, since no clinical trials have provided any specific data on patients with HER2 discordance (4), the guidelines 
for treatment and recommended therapies are still under exploration.

In this case report, we will share our experience of treating an advanced refractory breast cancer patient with HER2 discordance. This patient was first diagnosed as Luminal B-like HER2+, and her metastatic site was found to be Luminal B-like HER2-. The patient was prescribed palbociclib and exemestane, and as of April, 2020, her progression-free survival (PFS) had reached 14 months. There is currently a lack of clinical data on the efficacy of cyclin-dependent kinase inhibitors (CDKIs) in hormone receptor-positive breast cancer with HER2 discordance, but we believe this report will provide a basis for further research. We present the following case in accordance with the CARE reporting checklist (available at http://dx.doi. org/10.21037/tbcr-20-3).

\section{Case presentation}

In December 2018, a 40-year-old woman came to our hospital for further treatment for her advanced refractory breast cancer. The patient was first diagnosed with breast cancer in May, 2015, after which she received right breast radical mastectomy plus right axillary sentinel lymph node dissection on June 1, 2015. Pathological examination of the surgical sample identified the component as invasive ductal carcinoma with partial ductal carcinoma in situ. The Scarff-Bloom-Richardson grade was II. The tumor measured $2.5 \mathrm{~cm} \times 2 \mathrm{~cm} \times 1.5 \mathrm{~cm}$, and 7 metastases were found in 9 lymph nodes (pT2N2M0). The molecular breast cancer subtype was identified as Luminal B-like HER2+ by immunohistochemistry: estrogen receptor (ER) $50 \%+$, progesterone receptor (PR) 5\%+, HER2: 2+, FISH: +, Ki67: 30\%.

Consequently, the patient received 4 cycles of epirubicin and cyclophosphamide $\left(\mathrm{EC} \times 4-\mathrm{TH} \times 4\right.$ : epirubicin $90 \mathrm{mg} / \mathrm{m}^{2}$ plus cyclophosphamide $600 \mathrm{mg} / \mathrm{m}^{2}$ ), administered intravenously from June, 2015. This was followed by 4 cycles of docetaxel and trastuzumab (docetaxel $90 \mathrm{mg} / \mathrm{m}^{2}$ plus trastuzumab $8 \mathrm{mg} / \mathrm{kg}$ for the first dose, then $6 \mathrm{mg} / \mathrm{kg}$ after that), administered intravenously. Following the completion of docetaxel treatment in December, 2015, trastuzumab $(6 \mathrm{mg} / \mathrm{kg}$, administered intravenously in day 1 of each 21-day cycle) was administered until September, 2016, concluding 1 year of treatments. Adjuvant radiotherapy (unknown) was performed after the chemotherapy, and starting in January, 2016, the patient received endocrine therapy (daily letrozole, $2.5 \mathrm{mg} /$ day).
However, in December 2016, a new lump was found on the patient's right chest wall. Computed tomography (CT) detected regional costal pleural thickening on the right side of the sternum (Figure 1A). Cutaneous metastasis was confirmed by fine needle aspiration (FNA), but the immunohistochemistry failed due to inadequate sample cells. The patient was diagnosed as primary trastuzumab resistant and primary endocrine therapy resistant. As a result, lapatinib $(1,250 \mathrm{mg}$, administered orally once daily) plus Capecitabine $\left(1,000 \mathrm{mg} / \mathrm{m}^{2}\right.$, administered orally twice daily on days 1-14 of each 21-day cycle) was given as the first-line therapy. This therapy showed great effectiveness, and the patient's condition was evaluated as partial response according to the Respond Evaluation Criteria in Solid Tumors (RECIST) 1.1 system (Figure 1B). In February, 2018, the lump was observed to have grown in size (Figure 1C), and so lapatinib $(1,250 \mathrm{mg}$, administered orally once daily) plus paclitaxel $\left(120 \mathrm{mg} / \mathrm{m}^{2}\right.$, administered intravenously on days 1 and 15 of each 21-day cycle) was given for 6 cycles as a substitute. In July, 2018, lapatinib (1,250 mg, administered orally once daily) plus fulvestrant $(500 \mathrm{mg}$, administered intramuscularly on day 1 of each 28-day cycle, with an additional $500 \mathrm{mg}$ dose on day 15 of cycle 1) was initiated as maintenance treatment.

However, in September, 2018, another new lump was discovered on the patient's right chest wall (Figure 1D). Double-targeted anti-HER2 therapy (trastuzumab $8 \mathrm{mg} / \mathrm{kg}$ for the first dose, followed by $6 \mathrm{mg} / \mathrm{kg}$, administered intravenously on day 1 of each 21-day cycle; lapatinib $1,250 \mathrm{mg}$, administered orally once daily; capecitabine $1,000 \mathrm{mg} / \mathrm{m}^{2}$, administered orally twice daily on days $1-14$ of each 21 -day cycle) was employed; however, after 2 months, the size of the first lump had increased again, and the treatment was deemed to have failed (Figure 2A). Therefore, a cutaneous biopsy was performed, and immunohistochemistry showed the molecular subtype to be Luminal B-like HER2- (ER $75 \%+$, PR -, HER2: 2+, FISH: -). A next-generation sequencing (NGS) test performed on the patient's surgical samples revealed $E R B B 2$ amplification, ERBB3 alteration on T355I, PIK3CA alteration, MDM2 amplification, EPHB4 amplification, and TP53 alteration on R196 (Table 1). Considering the patient's complicated condition and the HER2 discordance, we decided on a therapy consisting of palbociclib and exemestane (palbociclib $125 \mathrm{mg}$, administered orally once daily on days 1-21 of each 28-day cycle; and exemestane $25 \mathrm{mg}$, administered orally once daily). The patient has been receiving this therapy since December, 2018, and was evaluated as having stable disease 

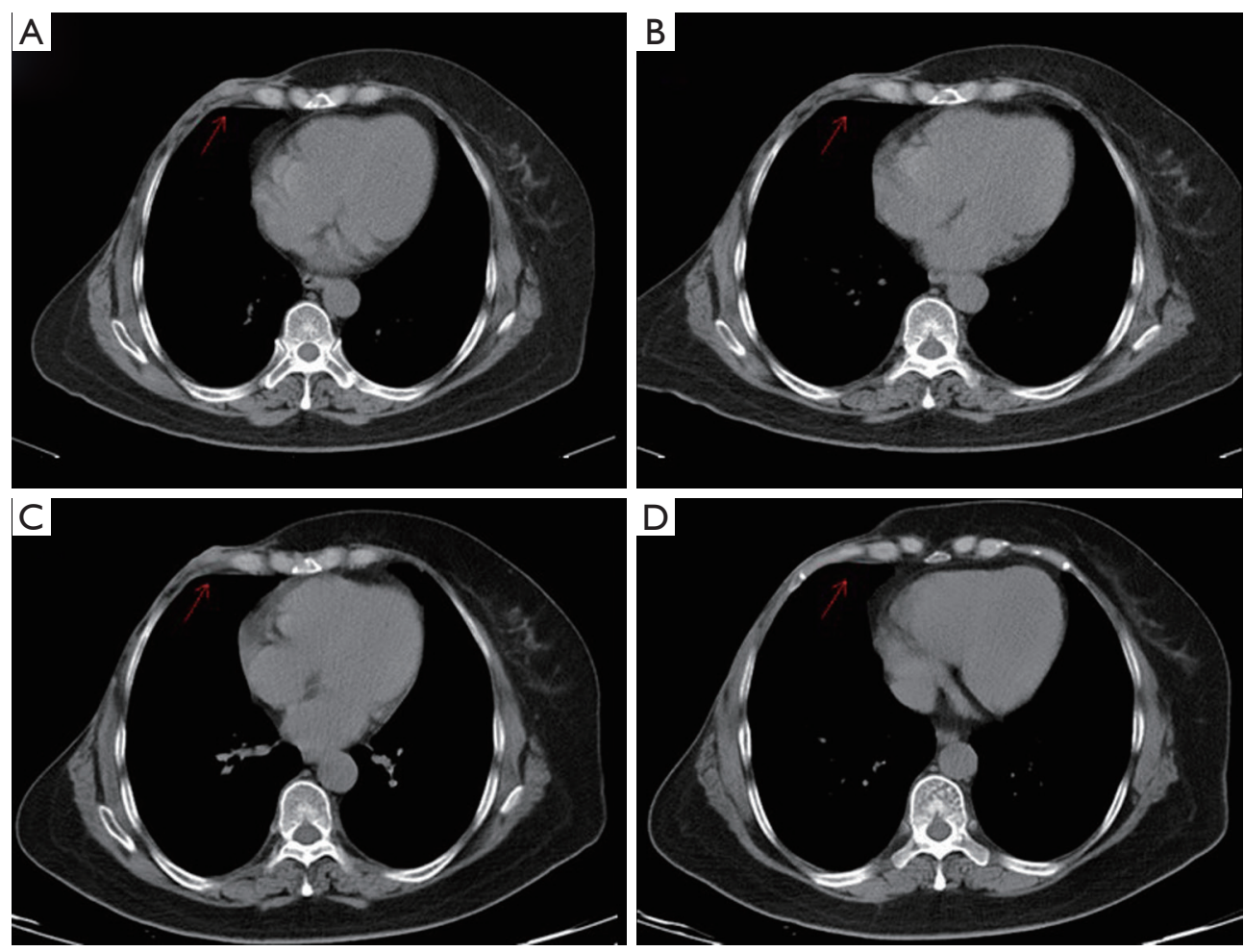

Figure 1 Computed tomography (CT) scan of previous metastases. (A) A metastatic site on the right chest wall was detected (red arrow) in December 2016; (B) the size of the metastasis was found to decrease after treatment with lapatinib plus capecitabine (red arrow). The effectiveness was evaluated as partial response according to the RECIST 1.1 system; (C) the metastatic site on the right chest wall was observed to have increased in size again in February, 2018 (red arrow); (D) a new metastatic site on the right chest wall was discovered in September, 2018 (red arrow), marking the failure of the second-line therapy.

according to the RECIST 1.1 system (Figure $2 B$ ). Several adverse effects were noted including neutropenia (grade II), and hypercholesterolemia, increased alanine transaminase (ALT), aspartate transaminase (AST), and alkaline phosphatase (ALP) (all grade I). Grade III increased gammaglutamyltransferase (GGT) was also observed. All of these adverse effects were evaluated according to the Common Terminology Criteria for Adverse Events (CTCAE) Version 5.0, and relevant responses and periodic reviews were taken to tackle them. The patient was followed up, and as of April, 2020, her progression-free survival (PFS) had reached 16 months (Figure 3). The patient has given her consent for the case report to be published.

\section{Discussion}

Breast cancer is heterogeneous at the molecular level. It can be classified into various subtypes, the molecular features of which, including the activation of hormone receptors, receptor discordance, and gene mutations, can often be observed (1). Here, we will analyze the inherent molecular heterogeneity of the patient in our report and the impact of HER2 discordance on treatment outcomes. This case report underscores the importance of pathological reassessment and standard treatments, as well as the potential role of palbociclib in treating hormone receptor-positive, HER2negative breast cancer with HER2 discordance.

This patient with primary Luminal B-like HER2+ breast cancer (pT2N2M0) received standard postoperative adjuvant chemotherapy, endocrine therapy, and radiotherapy according to Chinese Society of Clinical Oncology (CSCO) Guidelines on Breast Cancer. Three months after the end of adjuvant trastuzumab therapy, while patient was still receiving endocrine therapy, the disease was discovered to have progressed. Consequently, the patient was diagnosed as primary trastuzumab resistant and primary endocrine therapy resistant. Considering the meaningful role of NGS in precise medicine, we also performed an NGS test on 

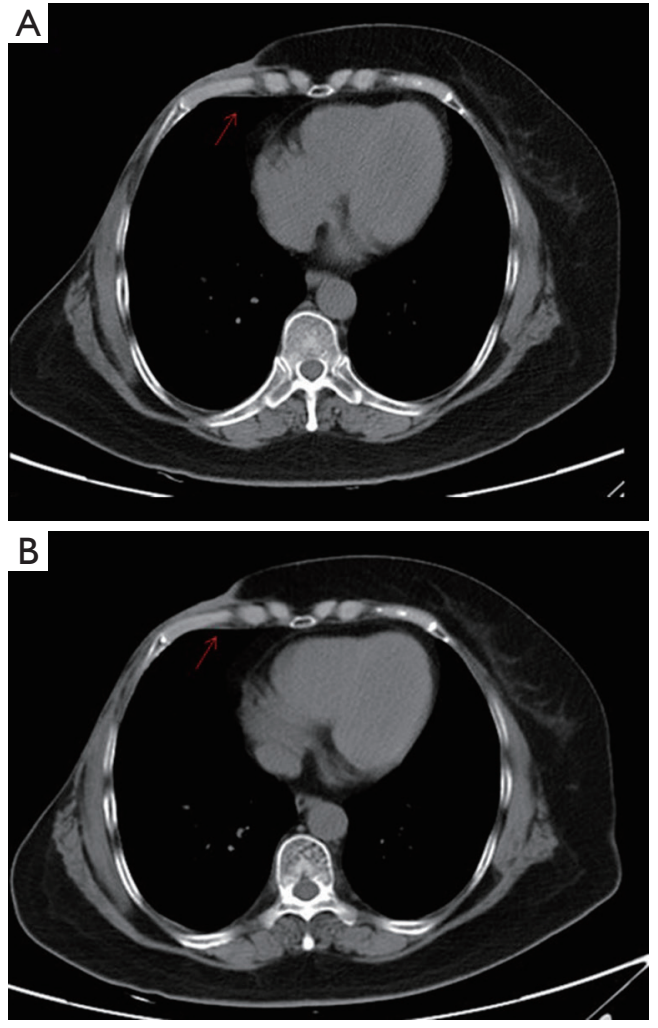

Figure 2 Computed tomography (CT) scan of the metastatic site before and after palbociclib and exemestane. (A) In December 2018, the metastasis on the right chest wall was found to have progressed, as the prescription of double-targeted anti-HER2 therapy (trastuzumab plus lapatinib plus capecitabine) had failed. (B) The thoracic CT scan (November, 2019) after receiving palbociclib and exemestane. The effectiveness of this therapy was evaluated as stable disease according to the RECIST 1.1 system, with no significant differences in the metastasis observed.

Table 1 The genomic findings of this patient's surgical samples

\begin{tabular}{lc}
\hline Gene & Mutations \\
\hline ERBB2 & Amplification-equivocal \\
ERBB3 & T355I \\
PIK3CA & $\mathrm{K} 111 \_1112>\mathrm{N}$ \\
MDM2 & Amplification \\
EPHB4 & Amplification \\
TP53 & R196 \\
\hline
\end{tabular}

ERBB2, Erb-B2 receptor tyrosine kinase 2; ERBB3, Erb-B2 receptor tyrosine kinase 3; PIK3CA, phosphatidylinositol-4,5bisphosphate 3-kinase catalytic subunit alpha; MDM2, MDM2 proto-oncogene; EPHB4, EPH receptor B4; TP53, tumor protein P53. her surgical samples and detected $E R B B 2$ amplification, ERBB 3 alteration on T355I, PIK3CA alteration, MDM2 amplification, EPHB4 amplification, and TP53 alteration on R196. Then, we analyzed the relationships between these mutations and trastuzumab resistance.

The mechanisms underlying trastuzumab resistance include impaired HER2 binding, activation of parallel or downstream pathways, enhancement of lipid metabolism, ER signaling, cell cycle regulation, and escape from antibody-dependent cellular cytoxicity (ADCC) (5). ERBB2 amplification, which is detected in $13 \%$ of breast cancer patients (6), promotes cancer cell proliferation and predicts sensitivity to HER2-targeted therapy (7). ERBB3 mutations have been found in up to $3 \%$ of breast invasive carcinomas (8). Overexpression of ERBB3 promotes HER2-HER3 heterodimer formation and results in resistance to trastuzumab (9). PIK3CA mutation, which has been reported in $25-40 \%$ of breast cancer cases (6), is associated with $P I 3 K / A k t / m T O R$ pathway activation (5). Both PIK3CA mutations and loss of phosphatase and tensin homolog (PTEN) have been reported to decrease the efficacy of trastuzumab in the treatment of metastatic breast cancers (10). Trastuzumab inhibits cancer cell proliferation mainly by blocking the $P I 3 K$ pathway (11), and this alteration helps cancer cells to bypass the inhibition and promotes their growth, differentiation, and survival (12). $M D M 2$ amplification, which is found in $3-7 \%$ breast carcinomas (6), decreases the status of Sirtuin 6, a tumor suppressor, and knockdown of $M D M 2$ resensitizes resistant breast cancer cells to trastuzumab (13). A recent study also showed that the inhibition of EPHB4 could enhance the sensitivity of breast cancer cells to anti-HER2 therapies like trastuzumab and lapatinib (14). TP53, one of the most common gene mutations in breast cancer, is a known tumor suppressor (15). Therapies targeting its mutations are still under research. Therefore, mutations of ERBB3, PIK3CA, $M D M 2$, and $E P H B 4$ were mainly responsible for this patient's trastuzumab resistance; however, the underlying mechanism needs further exploration. According to CSCO Guidelines on Breast Cancer, this patient was then treated with lapatinib plus capecitabine.

After the failure of the first-line therapy in February, 2018, the anti-HER2 therapy that followed achieved limited effectiveness; therefore, we decided to perform a biopsy for the second time to confirm the pathology of the metastasis. The molecular subtype was identified as Luminal B-like HER2-. The failure of anti-HER2 therapy was mostly attributable to the patient's loss of HER2 status (5), so we 


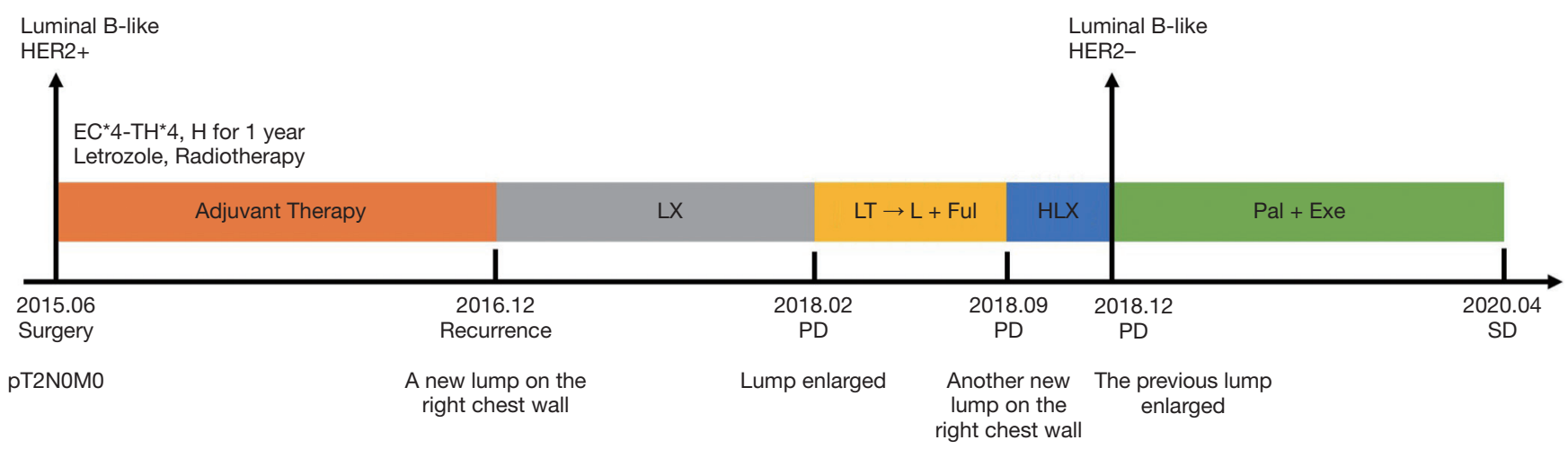

Figure 3 The patient's treatment timeline. EC, epirubicin and cyclophosphamide; TH, docetaxel and trastuzumab; LX, lapatinib and capecitabine; LT, lapatinib and paclitaxel; L, lapatinib; Ful, fulvestrant; HLX, trastuzumab and lapatinib and capecitabine; Pal, palbociclib; Exe, exemestane; PD, progressive disease; SD, stable disease.

explored the HER2 disordance in breast cancer.

HER2 discordance has been reported between the primary tumor and the metastatic site as well as between the primary tumor and the remaining tumor after the neoadjuvant chemotherapy (16). The mechanisms of HER2 discordance are still unclear, and there are several possible explanations including the reliability of immunohistochemistry testing technology, tumor heterogeneity, and biologic evolution (4). In a retrospective study of 205 breast cancer patients with HER2 overexpression, reassessment of the metastatic site showed that $42 \%(n=86)$ of cases had changed from HER2 positive to negative (16). The study also found that HER2targeted therapy was associated with HER2 discordance (16); however, another retrospective study of 182 patients reported that the HER2 discordance rate was related to whether patients received chemotherapy or trastuzumab (17). A review of 41 studies (1,828 paired samples) also revealed that the median discordance rate of HER2/neu between the primary and metastatic sites is $10 \%$ (range $0-44 \%$, IQR 4-17\%) (4). Moreover, whether or not a difference exists between the treatment outcomes of patients with discordant HER2 and concordant HER2 is controversial. Some studies have reported that the overall survival (OS) of patients with HER2 discordance is shorter than that of patients with concordant HER2 $(17,18)$, but some other researchers have maintained that no such association exists $(16,19)$. Dieci et al. reported post-recurrence survival (PRS) to be worse in patients with the loss of expression in HER2 and hormone receptors (18); however, Ibrahim et al. argued that PRS was only associated with ER loss in metastatic sites (20). Furthermore, since no studies have compared the outcomes of patients with discordance of HER2 or other receptors whose treatments were based on the receptor expression of the primary site versus that of the metastatic site, the selection of treatments for receptor discordance is still being explored (4). HER2 discordance is not uncommon during the treatment of breast cancer patients and may influence treatment outcomes. Therefore, it is necessary to reassess the metastatic site in later line therapies.

Since the pathological reassessment of our patient proved that the breast cancer subtype had changed from Luminal B-like HER2 + to Luminal B-like HER2-, we followed the treatment guidelines for hormone receptorpositive breast cancers. Considering the disease progression had been slow and there had been no visceral crisis, the endocrine therapy was prioritized. Therefore, we employed a therapy consisting of palbociclib and exemestane. Cyclindependent kinase 4/6 inhibitors (CDKIs), combined with endocrine therapy, have been approved by the US Food and Drug Administration (FDA) for both first-line and second-line treatment of hormone receptor-positive, HER2-negative, advanced or metastatic breast cancer. According to the PALOMA-2 study, as the first-line therapy of ER-positive, HER2-negative advanced breast cancer, palbociclib-letrozole resulted in a significantly longer PFS than letrozole-placebo (24.8 vs. 14.5 months) (21). PALOMA-3 also reported that palbociclib plus fulvestrant could significantly improve the PFS of ER-positive, HER2negative metastatic breast cancer patients as a second-line therapy, irrespective of resistance to endocrine therapy, PIK3CA mutations, and hormone-receptor expression status (22). Furthermore, CDKIs also have an emerging role in HER2 positive breast cancers. Since the enhanced activation of the cyclin D1-CDK4/6 axis is involved with 
resistance to anti-HER2 therapy (5), dual therapy including CDKIs and trastuzumab or other HER2-targeted drugs has a promising role in HER2-positive breast cancers and is under investigation in several clinical trials, such as PATINA and monarcHER (23). PATRICIA [ClinicalTrials. gov identifier: NCT02448420] has been examining the effectiveness of trastuzumab and pablociclib \pm letrozole in postmenopausal women with advanced HER2 + breast cancer who have previously received chemotherapy and trastuzumab, and the preliminary data has shown this dual therapy to have great clinical benefits. Recently, another study also reported that dual HER2 blockade could induce a change from a HER2-enriched subtype to a low-proliferative Luminal A subtype, especially when the hormone receptor was positive, and that the sensitivity to CDKIs was enhanced (24).

To date, there has been no clinical data on the use of CDKIs in patients with HER2 discordance. Considering the significant effectiveness of CDKIs in hormone receptorpositive breast cancers, and the enhanced sensitivity of cancer cells with HER2 discordance to CDKIs (24), we hypothized that CDKIs would benefit hormone receptorpositive breast cancer patients with HER2 discordance. As of April, 2020, this therapy had shown great effectiveness in our patient, whose PFS had reached 16 months.

The limitation of this report is that we still need an NGS test on the recurrent lump; this may help us understand the underlying relationship between CDKIs and HER2 discordance. More data about the clinical benefits of CDKIs in patients with HER2 discordance are also needed.

\section{Conclusions}

This case report focused on the role of CDKIs in treating hormone receptor-positive, HER2-negative breast cancer patients with HER2 discordance. HER2 discordance between the primary tumor and the metastatic site, as well as between the primary tumor and the remaining tumor, is often observed after neoadjuvant chemotherapy (16). The mechanism underlying this remains unclear, nor have a recommended therapy or guidelines been established for these patients. This case emphasizes the importance of the standard treatment and precise treatment, and enhances our awareness of pathological reassessment of metastases. Moreover, it also provides a novel regimen for HER2 discordance and deepens our insight into the role of CDKIs in breast cancer endocrine therapy. The relationship between CDKIs and HER2 discordance needs to be studied further, and we believe this case report will provide support for future research.

\section{Acknowledgments}

Funding: This case report was financially supported by the National Key Research and Development Program of China (ZDZX2017ZL-01), High-level Innovation Team of Nanjing Medical University (JX102GSP201727), Wu Jieping Foundation (320.6750.17006), Key Medical Talents (ZDRCA2016023), 333 Project of Jiangsu Province (BRA2017534 and BRA2015470), The Collaborative Innovation Center for Tumor Individualization Focuses on Open Topics (JX21817902/008) and Project of China Key Research and Development Program Precision Medicine Research (2016YFC0905901).

\section{Footnote}

Reporting Checklist: The authors have completed the CARE checklist. Available at http://dx.doi.org/10.21037/tbcr-20-3

Conflicts of Interest: All authors have completed the ICMJE uniform disclosure form (available at http://dx.doi. org/10.21037/tbcr-20-3). YY serves as an unpaid editorial board member of Translational Breast Cancer Research from Mar 2020 to Feb 2022. The other authors have no conflicts of interest to declare.

Ethical Statement: The authors are accountable for all aspects of the work in ensuring that questions related to the accuracy or integrity of any part of the work are appropriately investigated and resolved. All procedures performed in studies involving human participants were in accordance with the ethical standards of the institutional and/or national research committee(s) and with the Helsinki Declaration (as revised in 2013). The patient has given her consent for the case report to be published.

Open Access Statement: This is an Open Access article distributed in accordance with the Creative Commons Attribution-NonCommercial-NoDerivs 4.0 International License (CC BY-NC-ND 4.0), which permits the noncommercial replication and distribution of the article with the strict proviso that no changes or edits are made and the original work is properly cited (including links to both the formal publication through the relevant DOI and the license). See: https://creativecommons.org/licenses/by-nc-nd/4.0/. 


\section{References}

1. Harbeck N, Penault-Llorca F, Cortes J, et al. Breast cancer. Nat Rev Dis Primers 2019;5:66.

2. Van Poznak C, Somerfield MR, Bast RC, et al. Use of Biomarkers to Guide Decisions on Systemic Therapy for Women With Metastatic Breast Cancer: American Society of Clinical Oncology Clinical Practice Guideline. J Clin Oncol 2015;33:2695-704.

3. Lindström LS, Karlsson E, Wilking UM, et al. Clinically used breast cancer markers such as estrogen receptor, progesterone receptor, and human epidermal growth factor receptor 2 are unstable throughout tumor progression. J Clin Oncol 2012;30:2601-8.

4. Yeung C, Hilton J, Clemons M, et al. Estrogen, progesterone, and HER2/neu receptor discordance between primary and metastatic breast tumours-a review. Cancer Metastasis Rev 2016;35:427-37.

5. Vernieri C, Milano M, Brambilla M, et al. Resistance mechanisms to anti-HER2 therapies in HER2-positive breast cancer: Current knowledge, new research directions and therapeutic perspectives. Crit Rev Oncol Hematol 2019;139:53-66.

6. Cancer Genome Atlas Network. Comprehensive molecular portraits of human breast tumours. Nature 2012;490:61-70.

7. Higgins MJ, Baselga J. Targeted therapies for breast cancer. J Clin Invest 2011;121:3797-803.

8. Pereira B, Chin SF, Rueda OM, et al. The somatic mutation profiles of 2,433 breast cancers refines their genomic and transcriptomic landscapes. Nat Commun 2016;7:11479.

9. Sergina NV, Rausch M, Wang D, et al. Escape from HERfamily tyrosine kinase inhibitor therapy by the kinaseinactive HER3. Nature 2007;445:437-41.

10. Razis E, Bobos M, Kotoula V, et al. Evaluation of the association of PIK3CA mutations and PTEN loss with efficacy of trastuzumab therapy in metastatic breast cancer. Breast Cancer Res Treat 2011;128:447-56.

11. Derakhshani A, Rezaei Z, Safarpour H, et al. Overcoming trastuzumab resistance in HER2-positive breast cancer using combination therapy. J Cell Physiol 2020;235:3142-56.

12. Engelman JA. Targeting PI3K signalling in cancer: opportunities, challenges and limitations. Nat Rev Cancer 2009;9:550-62.

13. Thirumurthi U, Shen J, Xia W, et al. MDM2-mediated degradation of SIRT6 phosphorylated by AKT1 promotes tumorigenesis and trastuzumab resistance in breast cancer. Sci Signal 2014;7:ra71.

14. Ding J, Yao Y, Huang G, et al. Targeting the EphB4 receptor tyrosine kinase sensitizes HER2-positive breast cancer cells to Lapatinib. Cancer Lett 2020;475:53-64.

15. Uji K, Naoi Y, Kagara N, et al. Significance of TP53 mutations determined by next-generation "deep" sequencing in prognosis of estrogen receptor-positive breast cancer. Cancer Lett 2014;342:19-26.

16. Ignatov T, Gorbunow F, Eggemann H, et al. Loss of HER2 after HER2-targeted treatment. Breast Cancer Res Treat 2019;175:401-8.

17. Niikura N, Liu J, Hayashi N, et al. Loss of human epidermal growth factor receptor 2 (HER2) expression in metastatic sites of HER2-overexpressing primary breast tumors. J Clin Oncol 2012;30:593-9.

18. Dieci MV, Barbieri E, Piacentini F, et al. Discordance in receptor status between primary and recurrent breast cancer has a prognostic impact: a single-institution analysis. Ann Oncol 2013;24:101-8.

19. van Rooijen JM, de Munck L, de Graaf JC, et al. Limited human epidermal growth factor receptor 2 discordance in metastatic breast cancer patients treated with trastuzumab, a population based study. Eur J Cancer 2014;50:885-91.

20. Ibrahim T, Farolfi A, Scarpi E, et al. Hormonal receptor, human epidermal growth factor receptor-2, and Ki67 discordance between primary breast cancer and paired metastases: clinical impact. Oncology 2013;84:150-7.

21. Finn RS, Martin M, Rugo HS, et al. Palbociclib and Letrozole in Advanced Breast Cancer. N Engl J Med 2016;375:1925-36.

22. Cristofanilli M, Turner NC, Bondarenko I, et al. Fulvestrant plus palbociclib versus fulvestrant plus placebo for treatment of hormone-receptor-positive, HER2negative metastatic breast cancer that progressed on previous endocrine therapy (PALOMA-3): final analysis of the multicentre, double-blind, phase 3 randomised controlled trial. Lancet Oncol 2016;17:425-39.

23. O'Sullivan CC, Suman VJ, Goetz MP. The emerging role of CDK4/6i in HER2-positive breast cancer. Ther Adv Med Oncol 2019;11:1758835919887665.

24. Brasó-Maristany F, Griguolo G, Pascual T, et al. Phenotypic changes of HER2-positive breast cancer during and after dual HER2 blockade. Nat Commun 2020;11:385.

(English Language Editor: J. Reynolds)

doi: $10.21037 /$ tbcr-20-3

Cite this article as: Hua Y, Sun C, Yang F, Yang Y, Bao S, Yan X, Zeng T, Li W, Yin Y. An advanced refractory breast cancer patient with human epidermal growth factor receptor 2 discordance: a case report. Transl Breast Cancer Res 2020;1:19. 